\title{
Choquet Integral of Fuzzy-Number-Valued Functions: The Differentiability of the Primitive with respect to Fuzzy Measures and Choquet Integral Equations
}

\author{
Zengtai Gong, ${ }^{1}$ Li Chen, ${ }^{1,2}$ and Gang Duan ${ }^{3}$ \\ ${ }^{1}$ College of Mathematics and Statistics, Northwest Normal University, Lanzhou 730070, China \\ ${ }^{2}$ Department of Mathematics, Lanzhou City University, Lanzhou 730070, China \\ ${ }^{3}$ School of Traffic and Transportation, Lanzhou Jiaotong University, Lanzhou 730070, China \\ Correspondence should be addressed to Zengtai Gong; zt-gong@163.com
}

Received 25 January 2014; Accepted 22 May 2014; Published 9 June 2014

Academic Editor: Marco Donatelli

Copyright ( 2014 Zengtai Gong et al. This is an open access article distributed under the Creative Commons Attribution License, which permits unrestricted use, distribution, and reproduction in any medium, provided the original work is properly cited.

\begin{abstract}
This paper deals with the Choquet integral of fuzzy-number-valued functions based on the nonnegative real line. We firstly give the definitions and the characterizations of the Choquet integrals of interval-valued functions and fuzzy-number-valued functions based on the nonadditive measure. Furthermore, the operational schemes of above several classes of integrals on a discrete set are investigated which enable us to calculate Choquet integrals in some applications. Secondly, we give a representation of the Choquet integral of a nonnegative, continuous, and increasing fuzzy-number-valued function with respect to a fuzzy measure. In addition, in order to solve Choquet integral equations of fuzzy-number-valued functions, a concept of the Laplace transformation for the fuzzy-number-valued functions in the sense of Choquet integral is introduced. For distorted Lebesgue measures, it is shown that Choquet integral equations of fuzzy-number-valued functions can be solved by the Laplace transformation. Finally, an example is given to illustrate the main results at the end of the paper.
\end{abstract}

\section{Introduction}

The Choquet integral [1-4] with respect to a fuzzy measure was proposed by Murofushi and Sugeno. It was introduced by Choquet in potential theory with the concept of capacity. Then, it has been used for utility theory in the field of economic theory [5] and has been used for image processing, pattern recognition, information fusion, and data mining $[4,6-8]$ in the context of fuzzy measure theory [9-13].

The development of the theory of integral equations is closely linked to the study of mathematical physics problems. The integral equation has the extremely widespread application in the field of engineering and mechanics and so forth. The early history of integral equation goes back to the special integral equation studied by several mathematicians, such as Laplace, Fourier, Poisson, Abel, and Liouville in the late eighteenth and early nineteenth century. With the development of computing technology, the integral equation as one of the important foundations of engineering calculation has been widely and effectively used. Today, with physical problems becoming more and more complex, integral equation is becoming more and more useful.

Fuzzy integral and differential equations were discussed by many authors [14-16], which have been suggested as a way of modeling uncertain and incompletely specified systems. Sugeno has described carefully the representation of Choquet integral and Choquet integral equations of realvalued increasing functions, and some important conclusions have been obtained [17]. Unfortunately, it is not reasonable to assume that all data are real data before we elicit them from practical data. Sometimes, fuzzy data may exist, such as in pharmacological, financial, and sociological applications. Motivated by the above papers and related research works on this topic, the paper discusses the representation of Choquet integral and Choquet integral equations of increasing fuzzynumber-valued functions.

The rest of this study is organized as follows. In Section 2, we review some basic definitions of fuzzy measure and 
Choquet integrals of real-valued functions. Section 3 gives the definitions and the characterizations of the Choquet integrals of interval-valued functions and fuzzy-number-valued functions based on the nonadditive Sugeno measure. Furthermore, the operational schemes of above several classes of integrals on a discrete sets are investigated which enable us to calculate Choquet integrals in applications. Section 4 gives a representation of the Choquet integral of a nonnegative, continuous, and increasing fuzzy-number-valued function with respect to a fuzzy measure. In Section 5, in order to solve Choquet integral equations of fuzzy-number-valued functions, a concept of the Laplace transformation for the fuzzy-numbervalued functions in the sense of Choquet integral is introduced. For distorted Lebesgue measures, it is shown that Choquet integral equations of fuzzy-number-valued functions can be solved by the Laplace transformation. In addition, an example is given to illustrate the main results at the end of the paper. The paper ends with conclusions in Section 6.

\section{Preliminaries}

In this section, we will introduce some basic definitions about fuzzy measures, Choquet integral, and fuzzy numbers.

Definition 1 (see $[6,7,18-20])$. Let $X$ be a nonempty set and $\mathscr{A}$ a $\sigma$-algebra on $X$. A fuzzy measure on $X$ is a set function $\mu: \mathscr{A} \rightarrow[0, \infty)$ satisfying the following conditions:

(1) $\mu(\emptyset)=0$;

(2) $A \in X, B \in X, A \subset B$ implies $\mu(A) \leq \mu(B)$;

(3) In $X$, if $A_{1} \subset A_{2} \subset A_{3} \subset \cdots$, and $\bigcup_{n=1}^{\infty} A_{n} \in X$, then $\lim _{n \rightarrow \infty} \mu\left(A_{n}\right)=\mu\left(\bigcup_{n=1}^{\infty} A_{n}\right)$

(4) In $X$, if $A_{1} \supset A_{2} \supset A_{3} \supset \cdots$, and $\bigcap_{n=1}^{\infty} A_{n} \in X$, then $\lim _{n \rightarrow \infty} \mu\left(A_{n}\right)=\mu\left(\bigcap_{n=1}^{\infty} A_{n}\right)$.

$\mu$ is said to be lower semicontinuous if it satisfies the above conditions (1)-(3); $\mu$ is said to be upper semicontinuous if it satisfies the above conditions (1), (2), and (4); $\mu$ is said to be continuous if it satisfies the above conditions (1)-(4).

$(X, \mathscr{A}, \mu)$ is said to be a nonadditive measure space.

One can see that a fuzzy measure is a normal monotone set function which vanishes at the empty set. Furthermore, a fuzzy measure on $X$ is said to be

(i) additive if $\mu(A \cup B)=\mu(A)+\mu(B)$ for all disjoint subsets $A, B \in X$;

(ii) subadditive if $\mu(A \cup B) \leq \mu(A)+\mu(B)$ for all disjoint subsets $A, B \in X$;

(iii) superadditive if $\mu(A \cup B) \geq \mu(A)+\mu(B)$ for all disjoint subsets $A, B \in X$;

(iv) cardinality-based if for any $A \in X, \mu(A)$ depends only on the cardinality of $A$;

(v) a $0-1$ fuzzy measure if its range is $\{0,1\}$;

(vi) a $0-1$ possibility fuzzy measure focused on $A$, denoted by $\operatorname{Pos}_{A}$, if $\operatorname{Pos}_{A}(B)=1$ if and only if $A \cap B \neq \emptyset$, and $\operatorname{Pos}_{A}(B)=0$ otherwise; (vii) a $0-1$ necessity fuzzy measure focused on $A$, denoted by $\operatorname{Nec}_{A}$, if $\operatorname{Nec}_{A}(B)=1$ if and only if $B \subseteq A$, and $\operatorname{Nec}_{A}(B)=0$ otherwise.

Let $f: X \rightarrow(-\infty,+\infty)$ be a measurable function with respect to $\mathscr{A}$. That is, $f$ satisfies the condition

$$
f_{\alpha}=\{x \mid f(x) \geq \alpha\} \in \mathscr{A}
$$

for any $\alpha \in \mathbb{R}$.

Definition 2 (see [1]). Let $(X, \mathscr{A}, \mu)$ be a nonadditive measure space and $f$ a measurable function on $X$. The Choquet integral of a real-valued function $f: X \rightarrow(-\infty,+\infty)$ is defined as

$$
\text { (c) } \int_{X} f d \mu=\int_{-\infty}^{0}\left[\mu\left(f_{\alpha}\right)-\mu(X)\right] d \alpha+\int_{0}^{\infty} \mu\left(f_{\alpha}\right) d \alpha,
$$

if both of Riemann integrals exist and at least one of them has finite value.

Let $E \in \mathscr{A}$. Then Choquet integral of a nonnegative realvalued function $f: X \rightarrow[0,+\infty)$ is defined as

$$
\text { (c) } \int_{E} f d \mu=\int_{0}^{\infty} \mu\left(E \cap f_{\alpha}\right) d \alpha .
$$

Since $\mu\left(E \cap f_{\alpha}\right)$ is nonincreasing with respect to $\alpha$, the Choquet integral of real-valued function $f$ with respect to $\mu$ exists. If $(c) \int_{E} f d \mu<\infty$, then $f$ is said to be $C$-integrable with respect to $\mu$ on $X$. Choquet integral has the following properties [1].

(1) If $g \leq h$, then (c) $\int_{E} g d \mu \leq(c) \int_{E} h d \mu$.

(2) If $A \subset B$, then (c) $\int_{A} f d \mu \leq(c) \int_{B} f d \mu$.

(3) Let $\mu$ be lower semicontinuous. If $f_{n} \uparrow f$ a.e. in $E$, then $(c) \int_{E} f_{n} d \mu \uparrow(c) \int_{E} f d \mu$.

(4) Let $\mu$ be upper semicontinuous. If $f_{n} \downarrow f$ a.e. in $E$, and there exists a $C$-integrable function $g$ such that $f_{1} \leq g$, then $(c) \int_{E} f_{n} d \mu \downarrow$ (c) $\int_{E} f d \mu$.

$I\left(R^{+}\right)=\left\{r:[r, \bar{r}] \subset R^{+}\right\}$denotes the set of all interval numbers on $R^{+}$, where $R^{+}=[0,+\infty)$. With the definition from $\mathrm{Wu}$ et al. [21], interval numbers should satisfy the following basic operations:

(1) $r * p=[\underline{r} * \underline{p}, \bar{r} * \bar{p}](*$ denotes $+\vee \wedge)$;

(2) $k \cdot r=[k r, k \bar{r}],\left(k \in \mathbb{R}^{+}\right)$;

(3) $r \leq p \Leftrightarrow \underline{r} \leq \underline{p}, \bar{r} \leq \bar{p}$;

(4) $d(r, p)=\max \{|\underline{r}-p|,|\bar{r}-\bar{p}|\}$;

(5) If $d\left(r_{n}, r\right) \rightarrow 0$, then $r_{n} \rightarrow r$.

A fuzzy subset of $R$ is a function $u: R \rightarrow[0,1]$. For each fuzzy set $u$ defined as above, we denote by $u_{\lambda}=$ $\{x \in R: u(x) \geq \lambda\}$, for any $\lambda \in[0,1]$, its $\lambda$-level set. By suppu we denote the support of $u$; that is, the set $\{x \in R$ : $u(x)>0\}$. By $u_{0}$ we denote the closure of suppu; that is, $u_{0}=\overline{\{x \in R: u(x)>0\}}$. Let $E$ be the collection of all fuzzy sets of $R$. We call $u \in E$ a fuzzy number if it satisfies the following conditions [22]: 
(1) $u$ is normal; that is, there exists $x_{0} \in R$ such that $u\left(x_{0}\right)=1$;

(2) $u$ is fuzzy convex; that is, $u(\lambda x+(1-\lambda) y) \geq$ $\min \{u(x), u(y)\}$ for any $x, y \in R, 0 \leq \lambda \leq 1$

(3) $u$ is upper semicontinuous; that is, $u\left(x_{0}\right) \geq$ $\varlimsup_{k \rightarrow \infty} u\left(x_{k}\right)$ for any $x_{k} \in R(k=0,1,2, \ldots), x_{k} \rightarrow$ $x_{0}$;

(4) $u_{0}=\overline{\{x \in R: u(x)>0\}}$ is compact.

We denote the collection of all fuzzy numbers by $\widetilde{R}$.

$\widetilde{a}$ is said to be a nonnegative fuzzy number if supp, $\widetilde{a}=$ $\{x \in R \mid a(x)>0\} \subset R^{+}$. We can define the semiorder and the distance in space $\widetilde{R}[22]$. Let $\widetilde{a}, \widetilde{b} \in \widetilde{R}$. Then $\widetilde{a} \leq \widetilde{b}$ if $\underline{a}_{\lambda} \leq \underline{b}_{\lambda}$ and $\bar{a}_{\lambda} \leq \bar{b}_{\lambda}$ for $\lambda \in(0,1] ; \tilde{a}+\widetilde{b}=\widetilde{c}$ if $a_{\lambda}+b_{\lambda}=c_{\lambda}$ for $\lambda \in(0,1] ; D(\tilde{a}, \tilde{b})=\sup _{\lambda \in[0,1]} D\left(a_{\lambda}, b_{\lambda}\right)$ is the distance of $\widetilde{a}$ and $\widetilde{b}$. Let $\widetilde{a_{n}} \subset \widetilde{R}$. If $D\left(\widetilde{a_{n}}, \widetilde{a}\right) \rightarrow 0$, then $\widetilde{a_{n}} \rightarrow \widetilde{a}$.

$\widetilde{R^{+}}$denotes the collection of all nonnegative fuzzy numbers.

Lemma 3 (see [22]). Let $\widetilde{a} \in \widetilde{R}$. Then

(1) $a_{\lambda}$ is a nonempty, bounded, and closed interval for each $\lambda \in[0,1]$

(2) if $0 \leq \lambda_{1} \leq \lambda_{2} \leq 1$ then $a_{\lambda_{2}} \subset a_{\lambda_{1}}$;

(3) if $\lambda_{n} \geq 0$ and $\lambda_{n} \rightarrow \lambda(\lambda \in(0.1])$, then $\bigcap_{n=1}^{\infty} a_{\lambda_{n}}=a_{\lambda}$.

Conversely, there exists a $b_{\lambda} \subset R$ for each $\lambda \in(0,1]$, which satisfies the conditions (1)-(3); then there exists a unique fuzzy number $\tilde{a} \in \widetilde{R}$ such that $b_{\lambda}=a_{\lambda}, \lambda \in(0,1]$ and $a_{0}=\overline{\bigcup_{\lambda \in(0,1]} a_{\lambda}} \subset b_{0}$.

\section{Choquet Integrals of Interval-Valued Functions and Fuzzy-Number-Valued Functions Based on Nonadditive Measures}

In this section, we will give the definitions and the characterizations of the Choquet integrals of interval-valued functions and fuzzy-number-valued functions based on the nonadditive Sugeno measure. Furthermore, the operational schemes of above several classes of integrals on a discrete sets are investigated which enable us to calculate Choquet integrals in applications.

We first introduce the concept of the Choquet integrals for the interval-valued functions as follows.

Definition 4 (see [23]). An interval-valued function $F: X \rightarrow$ $I\left(R^{+}\right)$is said to be measurable if both $\underline{F}(x)$ and $\bar{F}(x)$ are measurable functions, where $F(x)=[F(x), \bar{F}(x)], F(x)$ is the left end point of interval $F(x)$ and $\bar{F}(x)$ is the right end point of interval $F(x)$.

Interval-valued function $F: X \rightarrow I\left(R^{+}\right)$is $C$-integrally bounded if there exists a Choquet integrable function $h$ : $X \rightarrow R^{+}$such that $|\dot{x}| \leq h(t)$ for every selection $\dot{x} \in F$.

We denote $P_{0}(X)=\{E \mid E \subset X$ and $E \neq \emptyset\}$. Let $(X, \mathscr{A}, \mu)$ be a measure space, $A, B \subset \mathscr{A}$, and let $F: A \rightarrow P_{0}(B)$ be a measurable set valued mapping. $f$ is said to be a measurable selection of $F$ if there exists a measurable mapping $f: A \rightarrow$ $B$ such that $f(x) \in F(x)$ for every $x \in A$.

Definition 5. Let $(X, \mathscr{A}, \mu)$ be a nonadditive measure space. Assume that $F: X \rightarrow I\left(R^{+}\right)$is measurable, $C$-integrally bounded interval-valued function, and $E \in \mathscr{A}$. F is said to be $C$-integrable if

$$
\text { (c) } \int_{E} F d \mu=:\left\{(c) \int_{E} g d \mu \mid g \in S_{F(x)}\right\}
$$

is a closed interval on $I\left(R^{+}\right)$, where

$$
\begin{aligned}
& S_{F(x)} \\
& \quad=\left\{g \mid g: X \longrightarrow R^{+} \text {is a measurable selection of } F(x)\right\} .
\end{aligned}
$$

Theorem 6. Let $(X, \mathscr{A}, \mu)$ be a nonadditive measure space. Suppose that $\mu$ is a fuzzy measure, $E \in \mathscr{A}$, and $F$ is nonnegative measurable, C-integrally bounded interval-valued function; then $F$ is $C$-integrable on $E$ and

$$
\text { (c) } \int_{E} F d \mu=\left[(c) \int_{E} \underline{F} d \mu,(c) \int_{E} \bar{F} d \mu\right] \text {. }
$$

Proof. Since $F$ is nonnegative measurable on $E, \underline{F}$ and $\bar{F}$ are measurable on $E$. Thus $F$ and $\bar{F}$ are two measurable selections of $F$. On the other hand, $F$ is $C$-integrally bounded; we have

$$
\underline{F} \leq h, \quad \bar{F} \leq h .
$$

By the properties of Choquet integral of real valued functions, we know that $F$ and $\bar{F}$ are $C$-integrable on $E$. Let $M \in$ (c) $\int_{E} F d \mu$. That is to say, there exists a measurable selection $g$ of $F$, such that $(c) \int_{E} g d \mu=M$. We can prove that $M \in$ $\left[(c) \int_{E} \underline{F} d \mu,(c) \int_{E} \bar{F} d \mu\right]$. Indeed, notice that $\underline{F} \leq g \leq \bar{F}$, and by the properties of Choquet integral of real-valued functions we have

$$
\text { (c) } \int_{E} \underline{F} d \mu \leq(c) \int_{E} g d \mu \leq(c) \int_{E} \bar{F} d \mu
$$

This follows that

$$
M \in\left[(c) \int_{E} \underline{F} d \mu,(c) \int_{E} \bar{F} d \mu\right] .
$$

Thus,

$$
\left\{(c) \int_{E} g d \mu \mid g \in S_{F(x)}\right\} \subset\left[(c) \int_{E} \underline{F} d \mu,(c) \int_{E} \bar{F} d \mu\right] .
$$

Conversely, we can show that

$$
\left[(c) \int_{E} \underline{F} d \mu,(c) \int_{E} \bar{F} d \mu\right] \subset(c) \int_{E} F d \mu .
$$

For measurable selections $p(x), m(x) \in F(x)$, we get that

$$
t p(x)+(1-t) m(x) \in F(x)
$$


is measurable selection of $F$ for any $0 \leq t \leq 1$. Therefore,

$$
\begin{aligned}
t \cdot(c) & \int_{E} p(x) d \mu+(1-t) \cdot(c) \int_{E} m(x) d \mu \\
& =(c) \int_{E}[t p(x)+(1-t) m(x)] d \mu \\
& \in\left\{(c) \int_{E} g d \mu \mid g \in S_{F(x)}\right\} .
\end{aligned}
$$

It implies that $(c) \int_{E} F d \mu=\left\{(c) \int_{E} g d \mu \mid g \in S_{F(x)}\right\}$ is a convex set. On the other hand, since $\underline{F}$ and $\bar{F}$ are two measurable selections of $F$, and

$$
\text { (c) } \int_{E} \underline{F} d \mu \in(c) \int_{E} F d \mu, \quad(c) \int_{E} \bar{F} d \mu \in(c) \int_{E} F d \mu,
$$

we have

$$
\left[(c) \int_{E} \underline{F} d \mu,(c) \int_{E} \bar{F} d \mu\right] \subset\left\{(c) \int_{E} g d \mu \mid g \in S_{F(x)}\right\} .
$$

Hence,

$$
\text { (c) } \int_{E} F d \mu=\left[(c) \int_{E} \underline{F} d g_{\mu},(c) \int_{E} \bar{F} d \mu\right] .
$$

That is, $F$ is $C$-integrable.

From the above theorem, we know that interval-valued function $F$ is $C$-integrable on $E$ if (c) $\int_{E} \underline{F} d \mu$ and (c) $\int_{E} \bar{F} d \mu$ exist and are bounded.

Next, we will introduce the concept of the Choquet integrals for the Fuzzy-number-valued functions as follows.

Fuzzy-number-valued function $\widetilde{F}: X \rightarrow \widetilde{R}^{+}$on $X$ is said to be measurable if $\underline{F}_{\lambda}$ and $\bar{F}_{\lambda}$ are measurable functions with respect to $x \in X$ for any $\lambda \in[0,1]$.

Fuzzy-number-valued function $\widetilde{F}: X \rightarrow \widetilde{R}^{+}$is said to be $C$-integrally bounded if there exists a Choquet integrable function $h: X \rightarrow R^{+}$such that $|\dot{x}| \leq h(t)$ for every selection $\dot{x} \in[\widetilde{F}(t)]_{0}$.

Definition 7. Let $(X, \mathscr{A}, \mu)$ be a nonadditive measure space. Assume that $E \in \mathscr{A}, \widetilde{F}: X \rightarrow \widetilde{R}^{+}$is measurable and $C$ integrally bounded function. $\widetilde{F}$ is said to be $F C$-integrable if

$$
\left\{\left[(c) \int_{E} \widetilde{F} d \mu\right]_{\lambda}=:\left\{(c) \int_{E} g d \mu \mid g \in S_{F_{\lambda}}\right\}, 0 \leq \lambda \leq 1\right\}
$$

determines a unique fuzzy number $\widetilde{a} \in \widetilde{R}^{+}$, which is denoted by $(c) \int_{E} \widetilde{F} d \mu=\widetilde{a}$, where $S_{F_{\lambda}}=\left\{g: X \rightarrow R^{+}, g \in F_{\lambda}\right.$ is a measurable selection of $F_{\lambda}$.

Theorem 8. Let $(X, \mathscr{A}, \mu)$ be a nonadditive measurable space. Assume $\mu$ is a continuous fuzzy measure, $E \in \mathscr{A}, \widetilde{F}: X \rightarrow \widetilde{R}^{+}$ is measurable and C-integrally bounded function; then $\widetilde{F}$ is FCintegrable on $E$ if and only if $\underline{F}_{\lambda}$ and $\bar{F}_{\lambda}$ are $C$-integrable on $E$ and

$$
\left[(c) \int_{E} \widetilde{F} d \mu\right]_{\lambda}=\left[(c) \int_{E} \underline{F}_{\lambda} d \mu,(c) \int_{E} \bar{F}_{\lambda} d \mu\right]
$$

for any $\lambda \in[0,1]$.
Proof. For the necessity, since $\widetilde{F}$ is measurable and $C$ integrally bounded function, $\underline{F}_{\lambda}$ and $\bar{F}_{\lambda}$ are measurable for every $\lambda \in[0,1]$ and there exists a Choquet integrable function $h(x)$ such that $0 \leq \underline{F}_{\lambda} \leq h(x), 0 \leq \bar{F}_{\lambda} \leq h(x)$, and then

$$
\text { (c) } \int_{E} \underline{F}_{\lambda} d \mu \leq(c) \int_{E} \bar{F}_{\lambda} d \mu \leq(c) \int_{E} h(x) d \mu<\infty .
$$

That is, $\underline{F}_{\lambda}, \bar{F}_{\lambda}$ are $C$-integrable and

$$
\left[(c) \int_{E} \tilde{F} d \mu\right]_{\lambda}=\left[(c) \int_{E} \underline{F}_{\lambda} d \mu,(c) \int_{E} \bar{F}_{\lambda} d \mu\right]
$$

for any $\lambda \in[0,1]$.

For the sufficiency, let $\widetilde{F}$ be a Fuzzy-number-valued function. Note that

$$
\left[(c) \int_{E} \widetilde{F} d \mu\right]_{\lambda}=\left[(c) \int_{E} \underline{F}_{\lambda} d \mu,(c) \int_{E} \bar{F}_{\lambda} d \mu\right] ;
$$

we need only to prove that the interval family

$$
\left\{\left[(c) \int_{E} \widetilde{F} d \mu\right]_{\lambda}=\left[(c) \int_{E} \underline{F}_{\lambda} d \mu,(c) \int_{E} \bar{F}_{\lambda} d \mu\right], \lambda \in[0,1]\right\}
$$

determines a unique fuzzy number. Indeed, the interval family

$$
\left\{\left[(c) \int_{E} \underline{F}_{\lambda} d \mu,(c) \int_{E} \bar{F}_{\lambda} d \mu\right]\right\}
$$

satisfies the conditions of Lemma 3.

(1) $\widetilde{F}$ is a measurable fuzzy-number-valued function; for each $\lambda \in[0,1]$, we have $\underline{F}_{\lambda}(x) \leq \bar{F}_{\lambda}(x)$, and therefore

$$
\text { (c) } \int_{E} \underline{F}_{\lambda} d \mu \leq(c) \int_{E} \bar{F}_{\lambda} d \mu \text {. }
$$

(2) Since $F_{\lambda_{2}} \subset F_{\lambda_{1}}$ for $0 \leq \lambda_{1} \leq \lambda_{2} \leq 1$, that is,

$$
\underline{F}_{\lambda_{1}}(x) \leq \underline{F}_{\lambda_{2}}(x), \quad \bar{F}_{\lambda_{1}}(x) \geq \bar{F}_{\lambda_{2}}(x),
$$

we have

$$
\begin{aligned}
& \text { (c) } \int_{E} \underline{F}_{\lambda_{1}} d \mu \leq(c) \int_{E} \underline{F}_{\lambda_{2}} d \mu, \\
& \text { (c) } \int_{E} \bar{F}_{\lambda_{1}} d \mu \geq(c) \int_{E} \bar{F}_{\lambda_{2}} d \mu, \\
& {\left[(c) \int_{E} \underline{F}_{\lambda_{1}} d \mu,(c) \int_{E} \bar{F}_{\lambda_{1}} d \mu\right]} \\
& \supset\left[(c) \int_{E} \underline{F}_{\lambda_{2}} d \mu,(c) \int_{E} \bar{F}_{\lambda_{2}} d \mu\right] .
\end{aligned}
$$

(3) For each $\lambda_{n} \uparrow \lambda \in(0,1], \bigcap_{n=1}^{\infty} F_{\lambda_{n}}(x)=F_{\lambda}(x)$, that is, $\lim _{n \rightarrow \infty} \underline{F}_{\lambda_{n}}=\underline{F}_{\lambda}, \lim _{n \rightarrow \infty} \bar{F}_{\lambda_{n}}=\bar{F}_{\lambda}$. It is easy to see 
that $\underline{F}_{\lambda_{1}} \leq \underline{F}_{\lambda_{n}} \leq \bar{F}_{\lambda_{n}} \leq \bar{F}_{\lambda_{1}}, \underline{F}_{\lambda_{1}}, \bar{F}_{\lambda_{1}}$ are integrable, and by the continuity of $\mu$, then

$$
\begin{array}{r}
\bigcap_{n=1}^{\infty}\left[(c) \int_{E} \underline{F}_{\lambda_{n}} d \mu,(c) \int_{E} \bar{F}_{\lambda_{n}} d \mu\right] \\
=\left[(c) \int_{E} \underline{F}_{\lambda} d \mu,(c) \int_{E} \bar{F}_{\lambda} d \mu\right] .
\end{array}
$$

In conclusion, there exists a unique fuzzy number $\widetilde{a} \in \widetilde{R}^{+}$ such that

$$
a_{\lambda}=\left[(c) \int_{E} \underline{F}_{\lambda} d \mu,(c) \int_{E} \bar{F}_{\lambda} d \mu\right] .
$$

Furthermore, we get that $\widetilde{F}$ is $F C$-integrable on $E$ and

$$
\left[(c) \int_{E} \widetilde{F} d \mu\right]_{\lambda}=\left[(c) \int_{E} \underline{F}_{\lambda} d \mu,(c) \int_{E} \bar{F}_{\lambda} d \mu\right] .
$$

In the last part of the section, we will investigate the operational schemes of above several classes of integrals on a discrete set.

Let $X=\left\{x_{1}, x_{2}, \ldots, x_{n}\right\}$ be a discrete set. Then we will give a new scheme to calculate the value of the Choquet integral.

Theorem 9 (see [8]). Let $f$ be a real-valued function on $X=$ $\left\{x_{1}, x_{2}, \ldots, x_{n}\right\}$. Then Choquet integral of $f$ with respect to a fuzzy measure $\mu$ on $X$ is given by

$$
\text { (c) } \int_{X} f d \mu=\sum_{i=1}^{n}\left[f\left(x_{i}^{\prime}\right)-f\left(x_{i-1}^{\prime}\right)\right] \mu\left(X_{i}^{\prime}\right),
$$

or equivalently, by

$$
\text { (c) } \int_{X} f d \mu=\sum_{i=1}^{n}\left[\mu\left(X_{i}^{\prime}\right)-\mu\left(X_{i+1}^{\prime}\right)\right] f\left(x_{i}^{\prime}\right),
$$

where $x_{1}^{\prime}, x_{2}^{\prime}, \ldots, x_{n}^{\prime}$ is a permutation of $x_{1}, x_{2}, \ldots, x_{n}$ such that $f\left(x_{0}^{\prime}\right) \leq f\left(x_{1}^{\prime}\right) \leq \cdots \leq f\left(x_{n}^{\prime}\right), f\left(x_{0}^{\prime}\right)=0, X_{i}^{\prime}=$ $\left\{x_{i}^{\prime}, x_{i+1}^{\prime}, \ldots, x_{n}^{\prime}\right\}, i=1,2, \ldots, n$, and $X_{n+1}^{\prime}=\emptyset$.

Theorem 10. Let $F$ be an interval-valued function on $X=$ $\left\{x_{1}, x_{2}, \ldots, x_{n}\right\}$. Then Choquet integral of $F$ with respect to a fuzzy measure $\mu$ on $X$ is given by

$$
\text { (c) } \int_{X} F d \mu=\sum_{i=1}^{n}\left[\mu\left(X_{i}^{\prime}\right)-\mu\left(X_{i+1}^{\prime}\right)\right] F\left(x_{i}^{\prime}\right)
$$

where $x_{1}^{\prime}, x_{2}^{\prime}, \ldots, x_{n}^{\prime}$ is a permutation of $x_{1}, x_{2}, \ldots, x_{n}$ such that $F\left(x_{0}^{\prime}\right) \leq F\left(x_{1}^{\prime}\right) \leq \cdots \leq F\left(x_{n}^{\prime}\right), F\left(x_{0}^{\prime}\right)=[0,0], X_{i}^{\prime}=$ $\left\{x_{i}^{\prime}, x_{i+1}^{\prime}, \ldots, x_{n}^{\prime}\right\}, i=1,2, \ldots, n$, and $X_{n+1}^{\prime}=\emptyset$.

Proof. Since $F$ is an interval-valued function on $X$, in view of Theorem 6, we have

$$
\text { (c) } \int_{X} F d \mu=\left[(c) \int_{X} \underline{F} d \mu,(c) \int_{X} \bar{F} d \mu\right] .
$$

Note that $\underline{F}$ and $\bar{F}$ are real-valued function on $X$, respectively. By Theorem 9 we get

$$
\begin{aligned}
\text { (c) } \int_{X} F d \mu & =\left[\sum_{i=1}^{n}\left[\mu\left(X_{i}^{\prime}\right)-\mu\left(X_{i+1}^{\prime}\right)\right] \underline{F}\left(x_{i}^{\prime}\right),\right. \\
\left.\sum_{i=1}^{n}\left[\mu\left(X_{i}^{\prime}\right)-\mu\left(X_{i+1}^{\prime}\right)\right] \bar{F}\left(x_{i}^{\prime}\right)\right] & =\sum_{i=1}^{n}\left[\mu\left(X_{i}^{\prime}\right)-\mu\left(X_{i+1}^{\prime}\right)\right] F\left(x_{i}^{\prime}\right),
\end{aligned}
$$

where $x_{1}^{\prime}, x_{2}^{\prime}, \ldots, x_{n}^{\prime}$ is a permutation of $x_{1}, x_{2}, \ldots, x_{n}$ such that $F\left(x_{0}^{\prime}\right) \leq F\left(x_{1}^{\prime}\right) \leq \cdots \leq F\left(x_{n}^{\prime}\right), F\left(x_{0}^{\prime}\right)=[0,0], X_{i}^{\prime}=$ $\left\{x_{i}^{\prime}, x_{i+1}^{\prime}, \ldots, x_{n}^{\prime}\right\}, i=1,2, \ldots, n$, and $X_{n+1}^{\prime}=\emptyset$.

Theorem 11. Let $\widetilde{F}$ be a fuzzy-number-valued function on $X=$ $\left\{x_{1}, x_{2}, \ldots, x_{n}\right\}$. Then Choquet integral of $\widetilde{F}$ with respect to a fuzzy measure $\mu$ on $X$ is given by

$$
\text { (c) } \int_{X} \widetilde{F} d \mu=\sum_{i=1}^{n}\left[\mu\left(X_{i}^{\prime}\right)-\mu\left(X_{i+1}^{\prime}\right)\right] \widetilde{F}\left(x_{i}^{\prime}\right),
$$

where $x_{1}^{\prime}, x_{2}^{\prime}, \ldots, x_{n}^{\prime}$ is a permutation of $x_{1}, x_{2}, \ldots, x_{n}$ such that $\widetilde{F}\left(x_{0}^{\prime}\right) \leq \widetilde{F}\left(x_{1}^{\prime}\right) \leq \cdots \leq \widetilde{F}\left(x_{n}^{\prime}\right), \widetilde{F}\left(x_{0}^{\prime}\right)=\widetilde{0}, X_{i}^{\prime}=$ $\left\{x_{i}^{\prime}, x_{i+1}^{\prime}, \ldots, x_{n}^{\prime}\right\}, i=1,2, \ldots, n$, and $X_{n+1}^{\prime}=\emptyset$.

Proof. Since $\widetilde{F}$ is a fuzzy-number-valued on $X$, in view of Theorem 8, we have

$$
\left[(c) \int_{X} \widetilde{F} d \mu\right]_{\lambda}=\left[(c) \int_{X} \underline{F}_{\lambda} d \mu,(c) \int_{X} \bar{F}_{\lambda} d \mu\right]
$$

for any $\lambda \in[0,1]$. By the semiorder in space $\widetilde{R}$ (i.e., let $\widetilde{a}, \widetilde{b} \in$ $\widetilde{R}$. Then $\widetilde{a} \leq \widetilde{b}$ if $\underline{a}_{\lambda} \leq \underline{b}_{\lambda}$ and $\left.\bar{a}_{\lambda} \leq \bar{b}_{\lambda}\right)$ and Theorem 10, there is a sequence $x_{1}^{\prime}, x_{2}^{\prime}, \ldots, x_{n}^{\prime}$ on $X$ such that $\widetilde{F}\left(x_{0}^{\prime}\right) \leq \widetilde{F}\left(x_{1}^{\prime}\right) \leq$ $\cdots \leq \widetilde{F}\left(x_{n}^{\prime}\right), \widetilde{F}\left(x_{0}^{\prime}\right)=\widetilde{0}, X_{i}^{\prime}=\left\{x_{i}^{\prime}, x_{i+1}^{\prime}, \ldots, x_{n}^{\prime}\right\}, i=1,2, \ldots, n$, $X_{n+1}^{\prime}=\emptyset$, and $x_{1}^{\prime}, x_{2}^{\prime}, \ldots, x_{n}^{\prime}$ is a permutation of $x_{1}, x_{2}, \ldots, x_{n}$. Consequently,

$$
\begin{aligned}
{\left[(c) \int_{X} \widetilde{F} d \mu\right]_{\lambda}=} & {\left[\sum_{i=1}^{n}\left[\mu\left(X_{i}^{\prime}\right)-\mu\left(X_{i+1}^{\prime}\right)\right] \underline{F}_{\lambda}\left(x_{i}^{\prime}\right),\right.} \\
& \left.\sum_{i=1}^{n}\left[\mu\left(X_{i}^{\prime}\right)-\mu\left(X_{i+1}^{\prime}\right)\right] \bar{F}_{\lambda}\left(x_{i}^{\prime}\right)\right]
\end{aligned}
$$

for any $\lambda \in[0,1]$. Therefore,

$$
\text { (c) } \int_{X} \widetilde{F} d \mu=\sum_{i=1}^{n}\left[\mu\left(X_{i}^{\prime}\right)-\mu\left(X_{i+1}^{\prime}\right)\right] \widetilde{F}\left(x_{i}^{\prime}\right) .
$$

\section{The Representation of Choquet Integral of Fuzzy-Number-Valued Functions}

Sugeno has described carefully the representation of Choquet integral of real-valued increasing functions, and some 
important conclusions have been obtained [17]. Motivated by this, we will discuss the representation of Choquet integral of fuzzy-number-valued functions in this section.

Definition 12. Fuzzy-number-valued function $\widetilde{F}: X \rightarrow \widetilde{R}^{+}$ is said to be continuous on $X$ if for every $x_{0} \in X$ there are

$$
\begin{aligned}
& \lim _{x \rightarrow x_{0}} \underline{F}_{\lambda}(x)=\underline{F}_{\lambda}\left(x_{0}\right), \\
& \lim _{x \rightarrow x_{0}} \bar{F}_{\lambda}(x)=\bar{F}_{\lambda}\left(x_{0}\right),
\end{aligned}
$$

where $\lambda \in[0,1]$.

Definition 13. Fuzzy-number-valued function $\widetilde{F}: X \rightarrow \widetilde{R}^{+}$ is said to be increasing on $X$ if for every $x_{1} \leq x_{2}$ there are $\underline{F}_{\lambda}\left(x_{1}\right) \leq \underline{F}_{\lambda}\left(x_{2}\right)$ and $\bar{F}_{\lambda}\left(x_{1}\right) \leq \bar{F}_{\lambda}\left(x_{2}\right)$, where $x_{1}, x_{2} \in X$ and $\lambda \in[0,1]$.

Let $\mathscr{F}^{+}$be a class of measurable, nonnegative, continuous, and increasing fuzzy-number-valued functions.

Let $v$ be a Lebesgue measure for $[a, b] \subset[0, \infty), v[a, b]=$ $b-a$.

Definition 14 (see [17]). Let $m: R^{+} \rightarrow R^{+}$be a continuous and increasing function and $m(0)=0$. A fuzzy measure $\mu_{m}$, a distorted Lebesgue measure, is defined by $\mu_{m}(\cdot)=m(\nu(\cdot))$.

Definition 15 (see [24]). A fuzzy-number-valued function $\widetilde{F}$ : $[a, b] \rightarrow \widetilde{R}$ is differentiable at $x \in[a, b]$ if there exist fuzzy number-valued functions $\widetilde{F}^{\prime}(x)$ such that

$$
\left[\widetilde{F}^{\prime}(x)\right]_{\lambda}=\left[\underline{F}_{\lambda}^{\prime}(x), \bar{F}_{\lambda}^{\prime}(x)\right]
$$

for every $\lambda \in[0,1]$, where $\widetilde{F}^{\prime}(x)$ is the fuzzy derivative of $\widetilde{F}(x)$.

Note that $\mu_{m}$ is induced from the Lebesgue measure $\nu$ by a monotone transformation, where $\mu_{m}([a, b])=m(\nu([a, b]))=$ $m(b-a)$. Apparently it loses additivity, unless $m(t)=t$, but reserves monotonicity. In what follows we assume that $m(t)$ is differentiable.

In this section, we consider the calculation of Choquet integrals. Let $\mu$ be a general fuzzy measure and consider $\mu([\tau, t])$ for a closed interval $[\tau, t]$; then $\mu([\tau, t])$ is decreasing for $\tau$ and increasing for $t$. Throughout the paper, we assume that the functions $m(t), \widetilde{F}(t)$ and $\widetilde{G}(t)$ are continuously differentiable. We also assume that $\mu([\tau, t])$ is continuously differentiable with respect to $\tau$ on $[0, t]$ for every $t>0$. In addition, we require the regularity condition that $\mu(\{t\})=0$ holds for every $t \geq 0$. We write $\mu^{\prime}([\tau, t])=(\partial / \partial \tau) \mu([\tau, t])$, where we note that $\mu^{\prime}([\tau, t]) \leq 0$ for $\tau \leq t$. If $\mu=\mu_{m}$ then $\mu^{\prime}([\tau, t])=-m^{\prime}(t-\tau)$ where $m^{\prime}(t)=d m(t) / d t$. First we consider a case that $\widetilde{F}(t)$ is strictly increasing.

Fuzzy-number-valued function $\widetilde{F}: X \rightarrow \widetilde{R}$ is said to be $L$-integrally bounded if there exists a Lebesgue integrable function $h: X \rightarrow R^{+}$such that $|\dot{x}| \leq h(t)$ for every section $\dot{x} \in[\widetilde{F}(t)]_{0}$.
Definition 16 (see [22]). Let fuzzy-number-valued functions $\widetilde{F}:[a,+\infty) \rightarrow \widetilde{R}$ be measurable and $L$-integrally bounded. $\widetilde{F}$ is called Kaleva-integrable if

$$
\begin{aligned}
& \left\{\left[\int_{a}^{+\infty} \widetilde{F}(t) d t\right]_{\lambda}\right. \\
& \left.\quad=:\left\{(L) \int_{a}^{+\infty} g(t) d t \mid g(t) \in S_{[\widetilde{F}(t)]_{\lambda}}\right\}, 0 \leq \lambda \leq 1\right\}
\end{aligned}
$$

determines a unique fuzzy number $\widetilde{a} \in \widetilde{R}$, which is denoted by $(K) \int_{a}^{+\infty} \widetilde{F}(t) d \mu=\widetilde{a}$, where $S_{[\widetilde{F}(t)]_{\lambda}}=\left\{g(t) \in[\widetilde{F}(t)]_{\lambda}\right.$ is a measurable selection\}.

Lemma 17 (see [22]). Let $\widetilde{F}:[a,+\infty) \rightarrow \widetilde{R}$ be measurable and $C$-integrally bounded fuzzy-number-valued function; then $\widetilde{F}$ is Kaleva integrable on $[a,+\infty)$ if and only if $\underline{F}_{\lambda}(t)$ and $\bar{F}_{\lambda}(t)$ are L-integrable on $[a,+\infty)$ and

$$
\left[(K) \int_{0}^{t} \widetilde{F}(\tau) d \tau\right]_{\lambda}=\left[(L) \int_{0}^{t} \underline{F}_{\lambda}(\tau) d \tau,(L) \int_{0}^{t} \bar{F}_{\lambda}(\tau) d \tau\right]
$$

for any $\lambda \in[0,1]$.

Theorem 18. Let $\widetilde{F}(t) \in \mathscr{F}^{+}$. Then $-\mu^{\prime}([\tau, t]) \widetilde{F}(\tau)$ is Kaleva integrable on $[0, t]$ and

$$
\begin{aligned}
& {\left[(K) \int_{0}^{t}\left[-\mu^{\prime}([\tau, t])\right] \widetilde{F}(\tau) d \tau\right]_{\lambda}} \\
& =\left[(L) \int_{0}^{t}\left[-\mu^{\prime}([\tau, t])\right] \underline{F}_{\lambda}(\tau) d \tau,\right. \\
& \left.(L) \int_{0}^{t}\left[-\mu^{\prime}([\tau, t])\right] \bar{F}_{\lambda}(\tau) d \tau\right] .
\end{aligned}
$$

Proof. Since $\widetilde{F}(t) \in \mathscr{F}^{+}$, for every $\lambda \in[0,1]$ we get that $\underline{F}_{\lambda}$ and $\bar{F}_{\lambda}$ are measurable, nonnegative, continuous, and increasing real-valued functions on $[0, t]$. It follows that $\underline{F}_{\lambda}$ and $\bar{F}_{\lambda}$ are $L$-integrable on $[0, t] . \mu([\tau, t])$ is $L$-integrable on $[0, t]$ as it is continuously differentiable with respect to $\tau$ on $[0, t]$ for every $t>0$. In view of Lemma $17,\left[-\mu^{\prime}([\tau, t])\right] \widetilde{F}(\tau)$ is Kaleva integrable on $[0, t]$ and

$$
\begin{aligned}
& {\left[(K) \int_{0}^{t}\left[-\mu^{\prime}([\tau, t])\right] \widetilde{F}(\tau) d \tau\right]_{\lambda}} \\
& =\left[(L) \int_{0}^{t}\left[-\mu^{\prime}([\tau, t])\right] \underline{F}_{\lambda}(\tau) d \tau,\right. \\
& \left.(L) \int_{0}^{t}\left[-\mu^{\prime}([\tau, t])\right] \bar{F}_{\lambda}(\tau) d \tau\right] .
\end{aligned}
$$

Lemma 19 (see [17]). Let $f(t)$ be a real strictly increasing function. Then the Choquet integral of $f$ with respect to $\mu$ on $[0, t]$ is represented as

$$
\text { (c) } \int_{[0, t]} f(\tau) d \mu(\tau)=-\int_{0}^{t} \mu^{\prime}([\tau, t]) f(\tau) d \tau .
$$


In particular, for $\mu=\mu_{m}$

(c) $\int_{[0, t]} f(\tau) d \mu_{m}(\tau)=\int_{0}^{t} m^{\prime}(t-\tau) f(\tau) d \tau$.

Lemma 20 (see [17]). Let $f(t)$ be a constant real-valued function: $f(t)=C, \forall t \in R^{+}=(0,+\infty)$. Then

$$
\text { (c) } \int_{[0, t]} f(\tau) d \mu(\tau)=-\int_{0}^{t} \mu^{\prime}([\tau, t]) C d \tau .
$$

In particular, for $\mu=\mu_{m}$

$$
\text { (c) } \int_{[0, t]} f(\tau) d \mu_{m}(\tau)=C m(t) .
$$

Lemma 21 (see [17]). Let $f(t)$ be a measurable, nonnegative, continuous, and increasing real-valued function. Then the Choquet integral of $f$ with respect to $\mu$ on $[0, t]$ is represented as

$$
\text { (c) } \begin{aligned}
\int_{[0, t]} f(\tau) d \mu(\tau) & =\int_{0}^{\infty} \mu(\{\tau \mid f(\tau) \geq r\} \cap[0, t]) d r \\
& =-\int_{0}^{t} \mu^{\prime}([\tau, t]) f(\tau) d \tau .
\end{aligned}
$$

In particular, for $\mu=\mu_{m}$

$$
\text { (c) } \begin{aligned}
\int_{[0, t]} f(\tau) d \mu_{m}(\tau) & =\int_{0}^{\infty} \mu(\{\tau \mid f(\tau) \geq r\} \cap[0, t]) d r \\
& =\int_{0}^{t} m^{\prime}(t-\tau) f(\tau) d \tau .
\end{aligned}
$$

Theorem 22. Let $\widetilde{F}(t)$ be a strictly increasing fuzzy-numbervalued function. Then the Choquet integral of $\widetilde{F}$ with respect to $\mu$ on $[0, t]$ is represented as

$$
\text { (c) } \int_{[0, t]} \widetilde{F}(\tau) d \mu(\tau)=(K) \int_{0}^{t}\left[-\mu^{\prime}([\tau, t])\right] \widetilde{F}(\tau) d \tau .
$$

In particular, for $\mu=\mu_{m}$

$$
\text { (c) } \int_{[0, t]} \widetilde{F}(\tau) d \mu_{m}(\tau)=(K) \int_{0}^{t} m^{\prime}(t-\tau) \widetilde{F}(\tau) d \tau .
$$

Proof. By Theorem 8, we have

$$
\begin{aligned}
& {\left[(c) \int_{[0, t]} \widetilde{F}(\tau) d \mu(\tau)\right]_{\lambda}} \\
& \quad=\left[(c) \int_{[0, t]} \underline{F}_{\lambda}(\tau) d \mu(\tau),(c) \int_{[0, t]} \bar{F}_{\lambda}(\tau) d \mu(\tau)\right]
\end{aligned}
$$

for any $\lambda \in[0,1]$. Since $\widetilde{F}(t)$ is strictly increasing, $\underline{F}_{\lambda}(t)$ and $\bar{F}_{\lambda}(t)$ are strictly increasing real-valued functions, respectively. In view of Lemma 19, we have

$$
\begin{aligned}
& \text { (c) } \int_{[0, t]} \underline{F}_{\lambda}(\tau) d \mu(\tau)=-\int_{0}^{t} \mu^{\prime}([\tau, t]) \underline{F}_{\lambda}(\tau) d \tau, \\
& \text { (c) } \int_{[0, t]} \bar{F}_{\lambda}(\tau) d \mu(\tau)=-\int_{0}^{t} \mu^{\prime}([\tau, t]) \bar{F}_{\lambda}(\tau) d \tau .
\end{aligned}
$$

It follows that

$$
\begin{aligned}
& {\left[(c) \int_{[0, t]} \widetilde{F}(\tau) d \mu(\tau)\right]_{\lambda}} \\
& \quad=\left[-\int_{0}^{t} \mu^{\prime}([\tau, t]) \underline{F}_{\lambda}(\tau) d \tau,-\int_{0}^{t} \mu^{\prime}([\tau, t]) \bar{F}_{\lambda}(\tau) d \tau\right] .
\end{aligned}
$$

On the other hand,

$$
\begin{aligned}
\int_{0}^{t} & {\left[-\mu^{\prime}([\tau, t])\right] \widetilde{F}_{\lambda}(\tau) d \tau } \\
& =\int_{0}^{t}\left[-\mu^{\prime}([\tau, t])\right]\left[\underline{F}_{\lambda}(\tau), \bar{F}_{\lambda}(\tau)\right] d \tau \\
& =\left[-\int_{0}^{t} \mu^{\prime}([\tau, t]) \underline{F}_{\lambda}(\tau) d \tau,-\int_{0}^{t} \mu^{\prime}([\tau, t]) \bar{F}_{\lambda}(\tau) d \tau\right] .
\end{aligned}
$$

Therefore,

$$
\left[(c) \int_{[0, t]} \widetilde{F}(\tau) d \mu(\tau)\right]_{\lambda}=\int_{0}^{t}\left[-\mu^{\prime}([\tau, t])\right] \widetilde{F}_{\lambda}(\tau) d \tau .
$$

By the arbitrary of $\lambda$, we have

$$
\text { (c) } \int_{[0, t]} \widetilde{F}(\tau) d \mu(\tau)=(K) \int_{0}^{t}\left[-\mu^{\prime}([\tau, t])\right] \widetilde{F}(\tau) d \tau .
$$

For $\mu=\mu_{m}$, we note that $-\mu^{\prime}([\tau, t])=m^{\prime}(t-\tau)$; hence,

$$
\text { (c) } \int_{[0, t]} \widetilde{F}(\tau) d \mu_{m}(\tau)=(K) \int_{0}^{t} m^{\prime}(t-\tau) \widetilde{F}(\tau) d \tau \text {. }
$$

Theorem 23. Let $\widetilde{F}(t)$ be a constant fuzzy-valued function; that is, $\widetilde{F}(t)=\widetilde{C}, \forall t \in R^{+}=(0,+\infty)$. Then

$$
\begin{aligned}
& \text { (c) } \int_{[0, t]} \widetilde{F}(\tau) d \mu(\tau) \\
& =(K) \int_{0}^{t}\left[-\mu^{\prime}([\tau, t])\right] \widetilde{C} d \tau=\widetilde{C} \mu([0, t]) .
\end{aligned}
$$

In particular, for $\mu=\mu_{m}$

$$
\text { (c) } \int_{[0, t]} \widetilde{F}(\tau) d \mu_{m}(\tau)=\widetilde{C} m(t) .
$$

Proof. Note that $\widetilde{C}=[\underline{C}, \bar{C}]$, where $\underline{C}$ and $\bar{C}$ are constant realvalued functions, respectively. In view of Lemma 20, we have
(c) $\int_{[0, t]} \underline{C} d \mu(\tau)=-\int_{0}^{t} \mu^{\prime}([\tau, t]) \underline{C} d \tau$,
(c) $\int_{[0, t]} \bar{C} d \mu(\tau)=-\int_{0}^{t} \mu^{\prime}([\tau, t]) \bar{C} d \tau$. 
The rest of the proof follows in exactly the same way as that of Theorem 22. Hence,

$$
\begin{aligned}
& \text { (c) } \int_{[0, t]} \widetilde{F}(\tau) d \mu(\tau) \\
& \quad=(K) \int_{0}^{t}\left[-\mu^{\prime}([\tau, t])\right] \widetilde{C} d \tau=\widetilde{C} \mu([0, t]) .
\end{aligned}
$$

For $\mu=\mu_{m}$, we obtain $\mu^{\prime}([\tau, t])=-m^{\prime}(t-\tau)$. It follows that

$$
\text { (c) } \begin{aligned}
\int_{[0, t]} \widetilde{C} d \mu(\tau) & =(K) \int_{0}^{t}\left[-\mu^{\prime}([\tau, t])\right] \widetilde{C} d \tau \\
& =\int_{0}^{t} m^{\prime}(t-\tau) \widetilde{C} d \tau \\
& =(K) \widetilde{C} \int_{0}^{t} m^{\prime}(t-\tau) d \tau=\widetilde{C} m(t),
\end{aligned}
$$

where $m(0)=0$.

Theorem 24. Let $\widetilde{F}(t) \in \mathscr{F}^{+}$. Then the Choquet integral of $\widetilde{F}$ with respect to $\mu$ on $[0, t]$ is represented as

(c) $\int_{[0, t]} \widetilde{F}(\tau) d \mu(\tau)=(K) \int_{0}^{t}\left[-\mu^{\prime}([\tau, t])\right] \widetilde{F}(\tau) d \tau$.

In particular, for $\mu=\mu_{m}$

(c) $\int_{[0, t]} \widetilde{F}(\tau) d \mu_{m}(\tau)=(K) \int_{0}^{t} m^{\prime}(t-\tau) \widetilde{F}(\tau) d \tau$.

Proof. The theorem has been proved when $\widetilde{F}(t)$ is a strictly increasing or constant fuzzy-number-valued function by Theorem 22 or Theorem 23. Without loss of generality, we consider a continuous and increasing function such that

$$
\widetilde{F}(t)= \begin{cases}\widetilde{F}_{1}(t), & 0 \leq t<t_{1}, \\ \widetilde{F}_{2}(t), & t_{1} \leq t<t_{2}, \\ \widetilde{F}_{3}(t), & t_{2} \leq t<\infty,\end{cases}
$$

where $\widetilde{F}_{1}(t)$ and $\widetilde{F}_{3}(t)$ are strictly increasing, $\widetilde{F}_{2}(t)$ is constant, and $\widetilde{F}_{1}\left(t_{1}\right)=\widetilde{F}_{2}(t)=\widetilde{F}_{3}\left(t_{2}\right)$.

(i) For $0 \leq t<t_{1}$

Note that $\widetilde{F}_{1}(t)$ is strictly increasing on $\left[0, t_{1}\right)$; from Theorem 22, we obtain

(c) $\int_{[0, t]} \widetilde{F}(\tau) d \mu(\tau)=(K) \int_{0}^{t}\left[-\mu^{\prime}([\tau, t])\right] \widetilde{F}_{1}(\tau) d \tau$.

(ii) For $t_{1} \leq t<t_{2}$

In view of the construction of $\widetilde{F}(t)$ on $[0, t]$, we have

$$
\begin{aligned}
(c) \int_{[0, t]} \widetilde{F}(\tau) d \mu(\tau)= & (c) \int_{\left[0, t_{1}\right]} \widetilde{F}_{1}(\tau) d \mu(\tau) \\
& +(c) \int_{\left[t_{1}, t\right]} \widetilde{F}_{2}(\tau) d \mu(\tau) .
\end{aligned}
$$

Since $\widetilde{F}_{1}(t)$ is strictly increasing on $\left[0, t_{1}\right)$, and $\widetilde{F}_{2}(t)$ is constant on $\left[t_{1}, t_{2}\right)$, by Theorems 22 and 23 ,

$$
\begin{aligned}
(c) \int_{[0, t]} \widetilde{F}(\tau) d \mu(\tau)= & (K) \int_{0}^{t_{1}}\left[-\mu^{\prime}([\tau, t])\right] \widetilde{F}_{1}(\tau) d \tau \\
& +(K) \int_{t_{1}}^{t}\left[-\mu^{\prime}([\tau, t])\right] \widetilde{F}_{2}(\tau) d \tau \\
= & (K) \int_{0}^{t}\left[-\mu^{\prime}([\tau, t])\right] \widetilde{F}(\tau) d \tau .
\end{aligned}
$$

(iii) For $t_{2} \leq t<\infty$

Since $\widetilde{F}_{1}(t)$ is strictly increasing on $\left[0, t_{1}\right), \widetilde{F}_{2}(t)$ is constant on $\left[t_{1}, t_{2}\right)$, and $\widetilde{F}_{3}(t)$ is strictly increasing on $\left[t_{1}, \infty\right)$, by Theorems 22 and 23, we have

$$
\begin{aligned}
& \text { (c) } \int_{[0, t]} \widetilde{F}(\tau) d \mu(\tau) \\
& =(c) \int_{\left[0, t_{1}\right]} \widetilde{F}_{1}(\tau) d \mu(\tau)+(c) \int_{\left[t_{1}, t_{2}\right]} \widetilde{F}_{2}(\tau) d \mu(\tau) \\
& \quad+(c) \int_{\left[t_{2}, t\right]} \widetilde{F}_{3}(\tau) d \mu(\tau) \\
& =(K) \int_{0}^{t_{1}}\left[-\mu^{\prime}([\tau, t])\right] \widetilde{F}_{1}(\tau) d \tau \\
& \quad+(K) \int_{t_{1}}^{t_{2}}\left[-\mu^{\prime}([\tau, t])\right] \widetilde{F}_{2}(\tau) d \tau \\
& \quad+(K) \int_{t_{2}}^{t}\left[-\mu^{\prime}([\tau, t])\right] \widetilde{F}_{3}(\tau) d \tau \\
& =(K) \int_{0}^{t}\left[-\mu^{\prime}([\tau, t])\right] \widetilde{F}^{\prime}(\tau) d \tau .
\end{aligned}
$$

For $\mu=\mu_{m}$, we obtain that

$$
\text { (c) } \int_{[0, t]} \widetilde{F}(\tau) d \mu_{m}(\tau)=(K) \int_{0}^{t} m^{\prime}(t-\tau) \widetilde{F}(\tau) d \tau \text {. }
$$

Remark 25. Note that Theorem 24 holds only for a continuous and increasing $\widetilde{F}$, but in the case $\mu_{m}=\nu$, it holds for any $\widetilde{F}$.

Remark 26. Let us consider the representation of the Choquet integral for a continuous case shown in Theorem 24 in relation with a discrete case. Now $[0, t]$ is transformed into the discrete set $\left\{x_{1}, x_{2}, \ldots, x_{n}\right\}$. Since $\widetilde{F}$ is an increasing fuzzynumber-valued function, we have $x_{i}=x_{i}^{\prime}, i=1,2, \ldots, n$ in Theorem 11. For the sake of simplicity, let $0=x_{0}<x_{1}<\cdots<$ 
$x_{n}=t, \widetilde{F}\left(x_{0}\right)=\widetilde{0}$ and $\left[x_{i}, t\right]=\left\{x_{i}, x_{i+1}, \ldots, x_{n}\right\}$. It follows that

$$
\text { (c) } \begin{aligned}
\int_{[0, t]} & \widetilde{F}(\tau) d \mu(\tau) \\
= & \sum_{i=1}^{n}\left[\mu\left(\left[x_{i}, t\right]\right)-\mu\left(\left[x_{i+1}, t\right]\right)\right] \widetilde{F}\left(x_{i}^{\prime}\right) \\
= & \sum_{i=1}^{n} \frac{-\left[\mu\left(\left[x_{i}, t\right]\right)-\mu\left(\left[x_{i+1}, t\right]\right)\right]}{\Delta x_{i}} \widetilde{F}\left(x_{i}^{\prime}\right) \Delta x_{i} \\
= & (K) \int_{0}^{t}\left[-\mu^{\prime}([\tau, t])\right] \widetilde{F}(\tau) d \tau .
\end{aligned}
$$

\section{Choquet Integral Equations of Fuzzy-Number-Valued Functions}

In this section, let us consider a Choquet integral equation based on Theorem 24 as shown below. Given continuous and increasing fuzzy-number-valued functions $\widetilde{G}(t)$ with $\widetilde{G}(0)=$ $\widetilde{0}$, let us find continuous and increasing fuzzy-number-valued functions $\widetilde{F}(t)$ such that

$$
\widetilde{G}(t)=(c) \int_{[0, t]} \widetilde{F}(\tau) d \mu_{m}(t)
$$

which is expressed as

$$
\widetilde{G}(t)=(K) \int_{0}^{t} m^{\prime}(t-\tau) \widetilde{F}(\tau) d \tau .
$$

Definition 27. Let $f(t)$ be a real-valued function defined on $[0,+\infty)$. The following

$$
\mathscr{F}(s)=\int_{0}^{+\infty} e^{-s t} f(t) d t
$$

is said to be the Laplace transformation of $f(t)$ if the infinite integral $\int_{0}^{+\infty} e^{-s t} f(t) d t$ is convergent with respect to the value of parameter $s$. We denote its Laplace transformation as $\mathscr{F}(s)=L[f(t)]$ and the inverse Laplace transformation as $f(t)=L^{-1}[\mathscr{F}(s)]$.

Definition 28. Let $\widetilde{F}(x, y)$ be a fuzzy-number-valued function defined on $[0,+\infty) \times[0,+\infty)$. $(K) \int_{0}^{\infty} \widetilde{F}(x, y) d x$ is said to be convergent with respect to the parameter $y$ if for every fixed $y \in[0,+\infty),(K) \int_{0}^{+\infty} \widetilde{F}(x, y) d x$ exists.

Definition 29. Let $\widetilde{F}(t)$ be a fuzzy-number-valued function defined on $[0,+\infty)$. The following

$$
\widetilde{\mathscr{F}}(s)=(K) \int_{[0,+\infty)} e^{-s t} \widetilde{F}(t) d t
$$

is said to be the Laplace transformation of $\widetilde{F}(t)$ if the Kaleva integral $\int_{0}^{\infty} e^{-s t} \widetilde{F}(t) d t$ is convergent with respect to the value of parameter $s$. We denote its Laplace transformation as $\widetilde{F}(s)=L[\widetilde{F}(t)]$ and the inverse Laplace transformation as $\widetilde{F}(t)=L^{-1}[\widetilde{\mathscr{F}}(s)]$.
Remark 30. In view of Theorem 8 , we have

$$
\widetilde{\mathscr{F}}_{\lambda}(s)=\left[\int_{0}^{+\infty} e^{-s t} \underline{F}_{\lambda}(t) d t, \int_{0}^{+\infty} e^{-s t} \bar{F}_{\lambda}(t) d t\right],
$$

for all $\lambda \in[0,1]$.

Theorem 31. Let $\widetilde{G}(t)=(c) \int_{[0, t]} \widetilde{F}(\tau) d \mu_{m}(t)$. Then

$$
\begin{aligned}
& \widetilde{\mathscr{G}}(s)=s \mathscr{M}(s) \widetilde{\mathscr{F}}(s), \\
& \widetilde{G}(s)=L^{-1}[s \mathscr{M}(s) \widetilde{\mathscr{F}}(s)],
\end{aligned}
$$

where $\widetilde{\mathscr{G}}(s)=L[\widetilde{G}(t)], \mathscr{M}(s)=L[m(t)]$, and $\widetilde{\mathscr{F}}(s)=L[\widetilde{F}(t)]$.

Proof. Since $\widetilde{G}(t)=(c) \int_{[0, t]} \widetilde{F}(\tau) d \mu_{m}(t)=(K) \int_{0}^{t} m^{\prime}(t-$ $\tau) \widetilde{F}(\tau) d \tau$, it follows easily from the condition that

$$
\begin{aligned}
\widetilde{\mathscr{G}}_{\lambda}(s)= & {\left[\int_{0}^{+\infty} e^{-s t} \underline{G}_{\lambda}(t) d t, \int_{0}^{+\infty} e^{-s t} \bar{G}_{\lambda}(t) d t\right] } \\
= & {\left[\int_{0}^{+\infty} e^{-s t} \int_{0}^{t} m^{\prime}(t-\tau) \underline{F}_{\lambda}(\tau) d \tau d t,\right.} \\
& \left.\int_{0}^{+\infty} e^{-s t} \int_{0}^{t} m^{\prime}(t-\tau) \bar{F}_{\lambda}(\tau) d \tau d t\right],
\end{aligned}
$$

for all $\lambda \in[0,1]$.

Notice that

$$
\begin{aligned}
& \int_{0}^{+\infty} e^{-s t} \int_{0}^{t} m^{\prime}(t-\tau) \underline{F}_{\lambda}(\tau) d \tau d t \\
& =\int_{0}^{+\infty} \int_{\tau}^{+\infty} e^{-s t} m^{\prime}(t-\tau) \underline{F}_{\lambda}(\tau) d t d \tau \\
& =\int_{0}^{+\infty} \underline{F}_{\lambda}(\tau) \int_{\tau}^{+\infty} e^{-s t} m^{\prime}(t-\tau) d t d \tau \\
& =\int_{0}^{+\infty} \underline{F}_{\lambda}(\tau)\left[\left.e^{-s t} m(t-\tau)\right|_{\tau} ^{+\infty}-\int_{\tau}^{+\infty} m(t-\tau) d e^{-s t}\right] d \tau \\
& =\int_{0}^{+\infty} \underline{F}_{\lambda}(\tau)\left[-\int_{\tau}^{+\infty} m(t-\tau)(-s) e^{-s t} d t\right] d \tau \\
& =s \int_{0}^{+\infty} \underline{F}_{\lambda}(\tau)\left[\int_{0}^{+\infty} m(t-\tau)(-s) e^{-s(t-\tau)} e^{-s \tau} d(t-\tau)\right] d \tau \\
& =s \int_{0}^{+\infty} \underline{F}_{\lambda}(\tau)\left[e^{-s \tau} \int_{0}^{+\infty} e^{-s(t-\tau)} m(t-\tau) d(t-\tau)\right] d \tau \\
& =s \int_{0}^{+\infty} \underline{F}_{\lambda}(\tau) e^{-s \tau} \mathscr{M}(s) d \tau \\
& =s \mathscr{M}(s) \int_{0}^{+\infty} e^{-s \tau} \underline{F}_{\lambda}(\tau) d \tau=s \mathscr{M}(s) \underline{F}_{\lambda}(s) .
\end{aligned}
$$

Following the same argument, we can prove that

$$
\int_{0}^{+\infty} e^{-s t} \int_{0}^{t} m^{\prime}(t-\tau) \bar{F}_{\lambda}(\tau) d \tau d t=s \mathscr{M}(s) \overline{\mathscr{F}}_{\lambda}(s) .
$$


Hence,

$$
\begin{aligned}
& \widetilde{\mathscr{G}}(s)=s \mathscr{M}(s) \widetilde{\mathscr{F}}(s), \\
& \widetilde{G}(s)=L^{-1}[s \mathscr{M}(s) \widetilde{\mathscr{F}}(s)] .
\end{aligned}
$$

Theorem 32. For continuous and increasing fuzzy-valued functions $\widetilde{G}(t)$ with $\widetilde{G}(0)=\widetilde{0}$,

$$
\widetilde{\mathscr{F}}(s)=\frac{\widetilde{\mathscr{G}}(s)}{s \mathscr{M}(s)}, \quad \widetilde{F}(s)=L^{-}\left[\frac{\widetilde{\mathscr{G}}(s)}{s \mathscr{M}(s)}\right],
$$

where $\widetilde{\mathscr{G}}(s)=L[\widetilde{G}(t)], \mathscr{M}(s)=L[m(t)]$, and $\widetilde{\mathscr{F}}(s)=L[\widetilde{F}(t)]$.

Proof. It follows easily from Theorem 31 that

$$
\begin{aligned}
& \widetilde{\mathscr{G}}(s)=s \mathscr{M}(s) \widetilde{\mathscr{F}}(s), \\
& \widetilde{G}(s)=L^{-1}[s \mathscr{M}(s) \widetilde{\mathscr{F}}(s)] .
\end{aligned}
$$

It is obvious that

$$
\begin{aligned}
& \widetilde{\mathscr{F}}(s)=\frac{\widetilde{\mathscr{G}}(s)}{s \mathscr{M}(s)}, \\
& \widetilde{F}(s)=L^{-1}\left[\frac{\widetilde{\mathscr{G}}(s)}{s \mathscr{M}(s)}\right],
\end{aligned}
$$

where $\widetilde{\mathscr{G}}(s)=L[\widetilde{G}(t)], \mathscr{M}(s)=L[m(t)]$, and $\widetilde{\mathscr{F}}(s)=L[\widetilde{F}(t)]$.

The Dirac delta function is defined by the properties

$$
\delta(t)= \begin{cases}0, & \text { for } t \neq 0, \\ \text { undefined, } & \text { at } t=0,\end{cases}
$$

and $\int_{-\infty}^{+\infty} \delta(t) d t=1$; that is, the function has unit area.

The Laplace transform of the Dirac Delta function $\delta(t)$ is shown as follows:

$$
L[\delta(t)]=\int_{0}^{+\infty} e^{-s t} \delta(t) d t=1 .
$$

So

$$
L^{-1}[1]=\delta(t)
$$

Example 33. Let $m(t)=t$ and let the membership function of $\widetilde{G}(t)$ be

$$
m_{\widetilde{G}(t)}(x)=\left\{\begin{array}{ll}
x, & x \in[0,1], \\
0, & x \notin[0,1],
\end{array} \quad t \leq 1 .\right.
$$

Then by Theorem 32 we can solve the Choquet integral equation

$$
\widetilde{G}(t)=(c) \int_{[0, t]} \widetilde{F}(\tau) d \mu_{m}(t) .
$$

Indeed, let $m(t)=t$; then $\mathscr{M}(s)=1 / s^{2}$. The $\lambda$-cut of $\widetilde{G}(t)$ is represented by interval: $\widetilde{G}_{\lambda}(t)=\left\{x \mid m_{\widetilde{G}(t)}(x) \geq \lambda\right\}=[\lambda, 1]$; that is $\underline{G}_{\lambda}(t)=\lambda, \bar{G}_{\lambda}(t)=1$. In view of Remark 30, we have $\widetilde{\mathscr{G}}_{\lambda}(s)=\left[\int_{0}^{+\infty} e^{-s t} \underline{G}_{\lambda}(t) d t, \int_{0}^{+\infty} e^{-s t} \bar{G}_{\lambda}(t) d t\right]=[\lambda / S, 1 / S]$. Then

$$
\begin{aligned}
& \underline{\mathscr{F}}_{\lambda}(s)=\frac{\mathscr{\mathscr { G }}_{\lambda}(s)}{s \mathscr{M}(s)}=\lambda, \\
& \overline{\mathscr{F}}_{\lambda}(s)=\frac{\overline{\mathscr{G}}_{\lambda}(s)}{s \mathscr{M}(s)}=1 .
\end{aligned}
$$

Furthermore,

$$
\begin{aligned}
& \underline{F}_{\lambda}(t)=L^{-1}\left(\underline{\mathscr{F}}_{\lambda}(s)\right)=\lambda \delta(t), \\
& \bar{F}_{\lambda}(t)=L^{-1}\left(\overline{\mathscr{F}}_{\lambda}(s)\right)=\delta(t) .
\end{aligned}
$$

Therefore, for every $\lambda \in[0,1]$

$$
F_{\lambda}(t)=\left[\underline{F}_{\lambda}(t), \bar{F}_{\lambda}(t)\right]=[\lambda \delta(t), \delta(t)] .
$$

Then we have the membership function of $\widetilde{F}(t)$ as

$$
m_{\widetilde{F}(t)}(x)= \begin{cases}\frac{x}{\delta(t)}, & x \in[0, \delta(t)], t=0, \\ 0, & \text { otherwise. }\end{cases}
$$

\section{Conclusions and Remarks}

In this paper, we have considered the Choquet integrals of fuzzy-number-valued functions based on the nonnegative real line. We have discussed the Choquet integrals of intervalvalued functions and fuzzy-number-valued functions based on nonadditive Sugeno measures and showed the representation theorem of them, respectively. We also gave the operational schemes of above several classes of integrals on discrete sets. Then we have given a representation of the Choquet integral of a nonnegative, continuous, and increasing fuzzy-number-valued function with respect to a fuzzy measure. In addition, in order to solve Choquet integral equations of fuzzy-number-valued function, a concept of the Laplace transformation for calculation has been introduced. For distorted Lebesgue measures, it was shown that Choquet integral equations of fuzzy-number-valued function could be solved by the Laplace transformation. Finally, we have given an example to illustrate the main result at the end of the paper.

\section{Conflict of Interests}

The authors declare that there is no conflict of interests regarding the publication of this paper.

\section{Acknowledgments}

The work is supported by the Natural Scientific Funds of China (61262022), the Natural Scientific Fund of Gansu Province of China (1208RJZA251), Lanzhou Jiaotong University Young Scientific Research Fund Project (2011020), the Fundamental Research Funds for Gansu Province Universities (no. 213060), and the Scientific Research Project of Northwest Normal University (no. NWNU-KJCXGC-03-61). 


\section{References}

[1] G. Choquet, "Theory of capacities," Annales de l'Institut Fourier, vol. 5, pp. 131-295, 1954.

[2] D. Denneberg, Non-Additive Measure and Integral, Kluwer Academic Publishers, Boston, Mass, USA, 1994.

[3] T. Murofushi, M. Sugeno, and M. Machida, "Non-monotonic fuzzy measures and the Choquet integral," Fuzzy Sets and Systems, vol. 64, no. 1, pp. 73-86, 1994.

[4] Z. Y. Wang, K.-S. Leung, M.-L. Wong, and J. Fang, "A new type of nonlinear integrals and the computational algorithm," Fuzzy Sets and Systems, vol. 112, no. 2, pp. 223-231, 2000.

[5] D. Schmeidler, "Subjective probability and expected utility without additivity, Econometrica, vol. 57, no. 3, pp. 571-587, 1989.

[6] M. Grabisch, " $k$-order additive discrete fuzzy measures and their representation," Fuzzy Sets and Systems, vol. 92, no. 2, pp. 167-189, 1997.

[7] M. Grabisch, I. Kojadinovic, and P. Meyer, "A review of methods for capacity identification in Choquet integral based multiattribute utility theory: applications of the Kappalab R package," European Journal of Operational Research, vol. 186, no. 2, pp. 766-785, 2008.

[8] K. Xu, Z. Wang, P. Heng, and K. Leung, "Classification by nonlinear integral projections," IEEE Transactions on Fuzzy Systems, vol. 11, no. 2, pp. 187-201, 2003.

[9] M. J. Bolanos, L. M. de Campos Ibañez, and A. Gonzalez Muñoz, "Convergence properties of the monotone expectation and its application to the extension of fuzzy measures," Fuzzy Sets and Systems, vol. 33, no. 2, pp. 201-212, 1989.

[10] T. Murofushi and M. Sugeno, "A theory of fuzzy measures: representations, the Choquet integral, and null sets," Journal of Mathematical Analysis and Applications, vol. 159, no. 2, pp. 532549, 1991.

[11] Z. Wang, "Convergence theorems for sequences of Choquet integrals," International Journal of General Systems, vol. 26, no. 1-2, pp. 133-143, 1997.

[12] Y. Ouyang and H. Zhang, "On the space of measurable functions and its topology determined by the Choquet integral," International Journal of Approximate Reasoning, vol. 52, no. 9, pp. 13551362, 2011.

[13] J. Kawabe, "The Choquet integral representability of comonotonically additive functionals in locally compact spaces," International Journal of Approximate Reasoning, vol. 54, no. 3, pp. 418-426, 2013.

[14] S. Abbasbandy, E. Babolian, and M. Alavi, "Numerical method for solving linear Fredholm fuzzy integral equations of the second kind," Chaos, Solitons \& Fractals, vol. 31, no. 1, pp. 138146, 2007.

[15] O. Kaleva, "Fuzzy differential equations," Fuzzy Sets and Systems, vol. 24, no. 3, pp. 301-317, 1987.

[16] J. Mordeson and W. Newman, "Fuzzy integral equations," Information Sciences, vol. 87, no. 4, pp. 215-229, 1995.

[17] M. Sugeno, "A note on derivatives of functions with respect to fuzzy measures," Fuzzy Sets and Systems, vol. 222, pp. 1-17, 2013.

[18] I. Kojadinovic, J.-L. Marichal, and M. Roubens, "An axiomatic approach to the definition of the entropy of a discrete Choquet capacity," Information Sciences, vol. 172, no. 1-2, pp. 131-153, 2005.

[19] E. Pap, Null-Additive Set Functions, vol. 337 of Mathematics and Its Applications, Kluwer Academic Publishers, London, UK, 1994.
[20] M. Sugeno, Theory of fuzzy integrals and its applications [Ph.D. dissertation], Tokyo Institute of Technology, Tokyo, Japan, 1974.

[21] C. X. Wu, D. L. Zhang, and C. M. Guo, "Fuzzy number fuzzy measures and fuzzy integrals," Fuzzy Sets and Systems, vol. 98, no. 3, pp. 355-360, 1998.

[22] C. X. Wu and M. Ma, The Foundament of Fuzzy Analysis, National Defense Press, Beijing, China, 1991, (Chinese).

[23] R. Yang, Z. Y. Wang, P. A. Heng, and K. S. Leung, "Fuzzy numbers and fuzzification of the Choquet integral," Fuzzy Sets and Systems, vol. 153, no. 1, pp. 95-113, 2005.

[24] S. Seikkala, "On the fuzzy initial value problem," Fuzzy Sets and Systems, vol. 24, no. 3, pp. 319-330, 1987. 


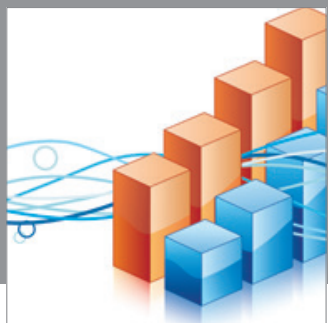

Advances in

Operations Research

mansans

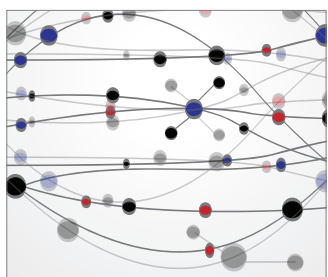

The Scientific World Journal
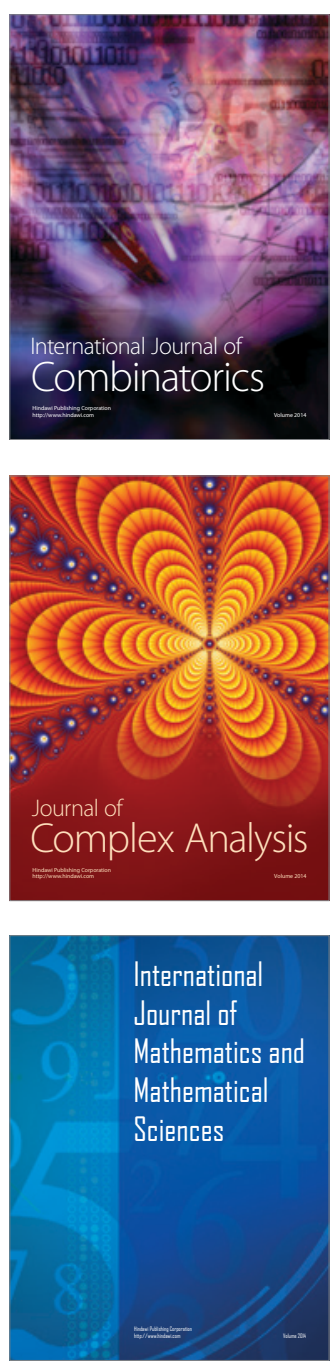
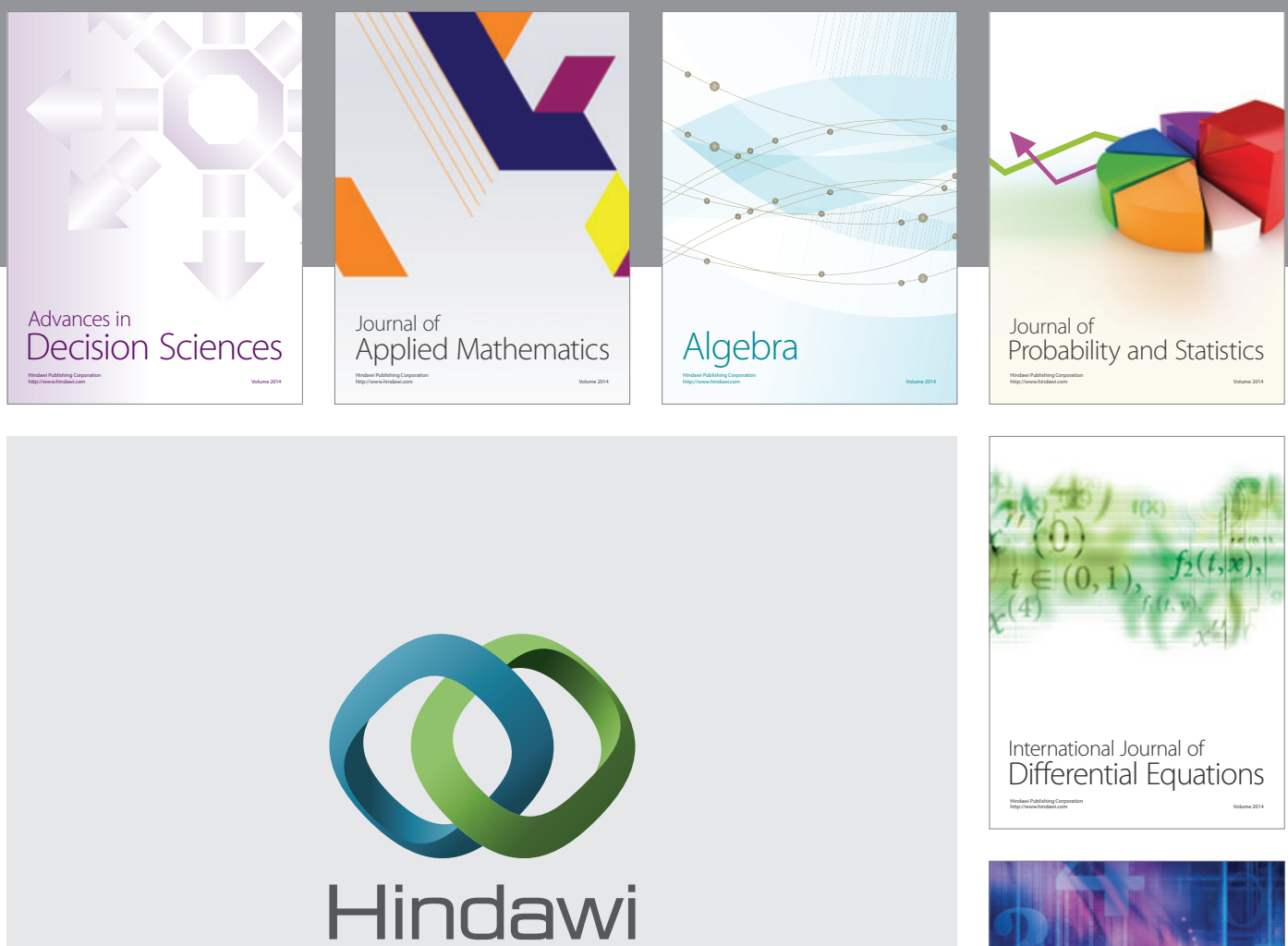

Submit your manuscripts at http://www.hindawi.com
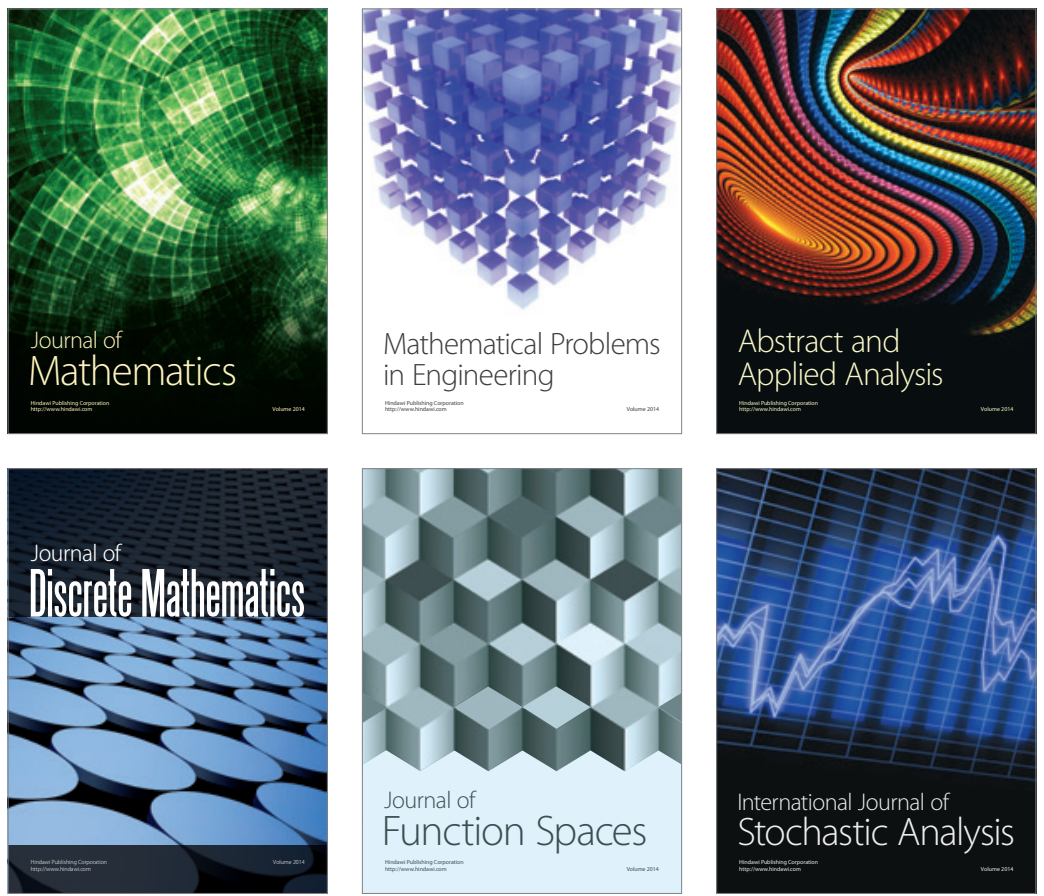

Journal of

Function Spaces

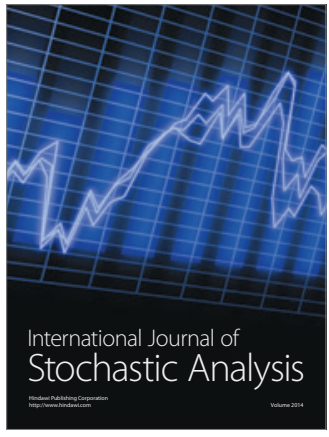

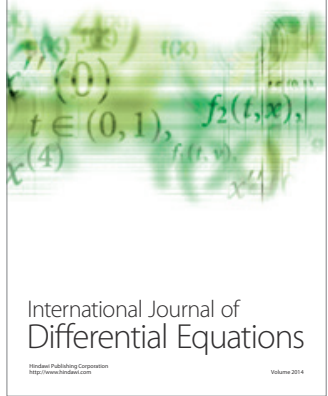
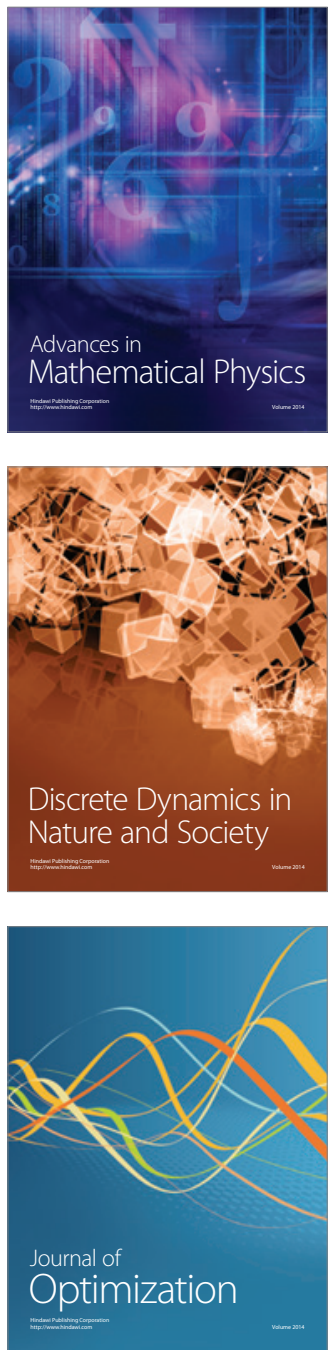